PDES, SUBMANIFOLDS AND

AFFINE DIFFERENTIAL GEOMETRY

BANACH CENTER PUBLICATIONS, VOLUME 57

INSTITUTE OF MATHEMATICS

POLISH ACADEMY OF SCIENCES

WARSZAWA 2002

\title{
CODAZZI STRUCTURES INDUCED BY MINIMAL AFFINE IMMERSIONS
}

\author{
HITOSHI FURUHATA \\ Department of Mathematics, Hokkaido University \\ Sapporo 060-0810, Japan \\ E-mail: furuhata@math.sci.hokudai.ac.jp
}

\begin{abstract}
We give a necessary and sufficient condition for a Codazzi structure to be realized as a minimal affine hypersurface or a minimal centroaffine immersion of codimension two.
\end{abstract}

Let $M$ be a simply-connected, oriented $C^{\infty}$-manifold of dimension $n \geq 2$. A pair $(\nabla, h)$ of a torsion-free affine connection and a semi-Riemannian metric on $M$ is called a Codazzi structure if the $(0,3)$-tensor field $\nabla h$ is symmetric. It can be observed that Codazzi structures play important roles in information geometry (e.g. [1]). In fact, they are often called statistical structures.

Let $R^{\nabla}$ be the curvature tensor field of $\nabla$ :

$$
R^{\nabla}(X, Y) Z:=\nabla_{X} \nabla_{Y} Z-\nabla_{Y} \nabla_{X} Z-\nabla_{[X, Y]} Z
$$

and $\operatorname{Ric}^{\nabla}$ the Ricci curvature tensor field of $\nabla$ :

$$
\operatorname{Ric}^{\nabla}(Y, Z):=\operatorname{tr}\left\{X \mapsto R^{\nabla}(X, Y) Z\right\} .
$$

It should be remarked that the scalar curvature is a primary invariant for a Codazzi structure:

$$
\operatorname{Scal}^{(\nabla, h)}:=\operatorname{tr}_{h}\left\{(X, Y) \mapsto \operatorname{Ric}^{\nabla}(X, Y)\right\}
$$

We briefly review affine immersion theory to fix the notation. Let $\mathbb{R}^{n+1}$ be the $(n+1)$ dimensional affine space equipped with the standard affine connection $D$ and the standard volume form Det.

Let $f: M \rightarrow \mathbb{R}^{n+1}$ be an affine immersion with Blaschke normal vector field $\xi$. By definition, the following hold: (1) At each point $x$ of $M$, the tangent space $T_{f(x)} \mathbb{R}^{n+1}$ is decomposed as $T_{f(x)} \mathbb{R}^{n+1}=f_{*} T_{x} M \oplus \mathbb{R} \xi_{x}$. For any vector fields $X, Y, Y_{j}$ on $M$, we write

$$
D_{X} f_{*} Y=f_{*} \nabla_{X} Y+h(X, Y) \xi, \quad D_{X} \xi=-f_{*} S X+\tau(X) \xi
$$

2000 Mathematics Subject Classification: Primary 53A15; Secondary 53C42.

Partially supported by the Inamori foundation.

The paper is in final form and no version of it will be published elsewhere. 


$$
\theta\left(Y_{1}, \ldots, Y_{n}\right):=\operatorname{Det}\left(f_{*} Y_{1}, \ldots, f_{*} Y_{n}, \xi\right),
$$

defining $\nabla, h, S, \tau$ and $\theta$. (2) $h$ gives rise to a semi-Riemannian metric on $M$. (3) $\nabla \theta=0$. (4) $\theta$ coincides with the volume form $\mathrm{Vol}_{h}$ determined by $h$, and is compatible with the orientation of $M$.

By the Codazzi equation, the pair $(\nabla, h)$ gives rise to a Codazzi structure on $M$ that is said to be induced by $f$.

An affine immersion $f: M \rightarrow \mathbb{R}^{n+1}$ is said to be minimal if the affine mean curvature $H:=1 / n \operatorname{tr} S$ vanishes identically. A minimal affine immersion is characterized as a critical point of a variational problem for the volume with respect to $\theta$.

Theorem 1. Let $M$ be a simply-connected, oriented $C^{\infty}$-manifold of dimension $n$, and $(\nabla, h)$ a Codazzi structure on $M$. A necessary and sufficient condition for $(\nabla, h)$ to be induced by a minimal affine immersion of $M$ into $\mathbb{R}^{n+1}$ is the following:

1. $\nabla \mathrm{Vol}_{h}=0$.

2. $(\nabla, h)$ is dual-projectively flat.

3. The scalar curvature of $(\nabla, h)$ identically vanishes.

The second condition means that the dual connection $\bar{\nabla}$ of $\nabla$ with respect to $h$ is projectively flat, which is equivalent to $\operatorname{tr}_{h}\left\{(Y, Z) \mapsto\left(\nabla_{Y} \operatorname{Ric}^{\nabla}\right)(Z, X)\right\}=0$ for any $X \in T_{x} M$ in the case that $\operatorname{dim} M=2$.

Theorem 1 directly follows from the generalized Radon theorem ([2], [4]). We should only remark that the Gauss equation: $R^{\nabla}(X, Y) Z=h(Y, Z) S X-h(X, Z) S Y$, implies $\operatorname{Scal}^{(\nabla, h)}=2 H$.

Returning to a review of affine immersion theory, we introduce centroaffine immersions of codimension two in the manner of [7]. Let $f: M \rightarrow \mathbb{R}^{n+2}$ be a centroaffine immersion with Blaschke normal vector field $\xi$. By definition, the following hold: (1) At each point $x$ of $M$, the tangent space $T_{f(x)} \mathbb{R}^{n+2}$ is decomposed as $T_{f(x)} \mathbb{R}^{n+2}=f_{*} T_{x} M \oplus \mathbb{R} \xi_{x} \oplus \mathbb{R} f(x)$. For any vector fields $X, Y, Y_{j}$ on $M$, we write

$$
\begin{gathered}
D_{X} f_{*} Y=f_{*} \nabla_{X} Y+h(X, Y) \xi+T(X, Y) f, \quad D_{X} \xi=-f_{*} S X+\tau(X) \xi+P(X) f, \\
\theta\left(Y_{1}, \ldots, Y_{n}\right):=\operatorname{Det}\left(f_{*} Y_{1}, \ldots, f_{*} Y_{n}, \xi, f\right),
\end{gathered}
$$

defining $\nabla, h, T, S, \tau, P$ and $\theta$. (2) $h$ gives rise to a semi-Riemannian metric on $M$. (3) $\nabla \theta=0$. (4) $\theta$ coincides with the volume form $\mathrm{Vol}_{h}$ determined by $h$, and is compatible with the orientation of $M$. (5) $\operatorname{tr}_{h}\{(X, Y) \mapsto T(X, Y)+h(S X, Y)\}=0$.

In the same fashion, a centroaffine immersion $f: M \rightarrow \mathbb{R}^{n+2}$ is said to be minimal if the affine mean curvature $H:=1 / n \operatorname{tr} S$ vanishes identically. A minimal centroaffine immersion is also characterized as a critical point of a certain variational problem ([3]).

TheOREM 2. Let $M$ be a simply-connected, oriented $C^{\infty}$-manifold of dimension $n$, and $(\nabla, h)$ a Codazzi structure on $M$. A necessary and sufficient condition for $(\nabla, h)$ to be induced by a minimal centroaffine immersion of $M$ into $\mathbb{R}^{n+2}$ is the following:

1. $\nabla \mathrm{Vol}_{h}=0$.

2. $(\nabla, h)$ is conformally-projectively flat.

3. The scalar curvature of $(\nabla, h)$ identically vanishes. 
The second condition means that there exist two functions $\phi$ and $\psi$ on $M$ such that the pair $(\widetilde{\nabla}, \tilde{h})$, defined by $\widetilde{\nabla}_{X} Y:=\nabla_{X} Y+d \phi(Y) X+d \phi(X) Y-h(X, Y) \operatorname{grad}_{h} \psi$ and $\widetilde{h}:=e^{\phi+\psi} h$, gives rise to a flat Codazzi structure. In the case that $\operatorname{dim} M \geq 4$, it is equivalent to the condition that the conformal-projective curvature tensor of $(\nabla, h)$, given by

$$
\begin{aligned}
\mathcal{W} K^{(\nabla, h)}(X, Y) Z:= & R^{\nabla}(X, Y) Z-\{n(n-1)\}^{-1} \operatorname{Scal}^{(\nabla, h)}\{h(Y, Z) X-h(X, Z) Y\} \\
& -\{n(n-2)\}^{-1} \\
& \cdot\left[h(Y, Z)\left\{\operatorname{Ric}^{(\nabla, h)}(X)+(n-1) \operatorname{Ric}^{(\bar{\nabla}, h)}(X)-\operatorname{Scal}^{(\nabla, h)} X\right\}\right. \\
& -h(X, Z)\left\{\operatorname{Ric}^{(\nabla, h)}(Y)+(n-1) \operatorname{Ric}^{(\bar{\nabla}, h)}(Y)-\operatorname{Scal}^{(\nabla, h)} Y\right\} \\
& +\left\{(n-1) \operatorname{Ric}^{\nabla}(Y, Z)+\operatorname{Ric}^{\bar{\nabla}}(Y, Z)-\operatorname{Scal}^{(\nabla, h)} h(Y, Z)\right\} X \\
& \left.-\left\{(n-1) \operatorname{Ric}^{\nabla}(X, Z)+\operatorname{Ric}^{\nabla}(X, Z)-\operatorname{Scal}^{(\nabla, h)} h(X, Z)\right\} Y\right],
\end{aligned}
$$

vanishes identically, where $\operatorname{Ric}^{(\nabla, h)}$ is the $(1,1)$-tensor field defined by $h\left(\operatorname{Ric}^{(\nabla, h)}(X), Y\right)$ $=\operatorname{Ric}^{\nabla}(X, Y)$.

Theorem 2 follows from the Matsuzoe-Kurose theorem ([6] and [5]), which is the counterpart of the generalized Radon theorem in the theory of centroaffine immersions of codimension two. We should only remark that the Gauss equation: $R^{\nabla}(X, Y) Z=$ $h(Y, Z) S X-h(X, Z) S Y-T(Y, Z) X+T(X, Z) Y$, together with the normalizing condition (5) implies $\operatorname{Scal}^{(\nabla, h)}=2 n(n-1) H$.

In a sense, we have given an intrinsic characterization for minimal affine immersions. This problem was pointed out by Opozda at the problem session of the conference at the Banach Center in 2000. The author wishes to express his gratitude to Professors Udo Simon and Barbara Opozda for organizing such an instructive and exciting meeting. The following problem is still open:

Problem 1. Give a necessary and sufficient condition for an equiaffine structure $(\nabla, \theta)$ to be realized as minimal affine immersions.

\section{References}

[1] S. Amari and H. Nagaoka, Methods of Information Geometry, Translations of Mathematical Monographs 191, Amer. Math. Soc., 2000.

[2] F. Dillen, K. Nomizu and L. Vrancken, Conjugate connections and Radon's theorem in affine differential geometry, Monatsh. Math. 109 (1990), 221-235.

[3] H. Furuhata, Minimal centroaffine immersions of codimension two, Bull. Belgian Math. Soc. 7 (2000), 125-134.

[4] S. Ivanov, On dual-projectively flat affine connections, J. Geom. 53 (1995), 89-99.

[5] T. Kurose, Conformal-projective geometry of statistical manifolds, preprint.

[6] H. Matsuzoe, On realization of conformally-projectively flat statistical manifolds and the divergences, Hokkaido Math. J. 27 (1998), 409-421.

[7] K. Nomizu and T. Sasaki, Centroaffine immersions of codimension two and projective hypersurface theory, Nagoya Math. J. 132 (1993), 63-90. 